\title{
Maestría en Docencia de las matemáticas
}

\author{
Titulo. Análisis sobre la transición del concepto de exponente natural \\ al concepto de exponente entero y racional en algunos textos escolares \\ de grado noveno.
}

Autor. PRIETO MONTENEGRO, Jeremy.

Palabras clave. Coherencia, Jerarquía, Convención Matemática.

\section{Descripción}

En este trabajo de grado se analizó la siguiente situación atendiendo a la pregunta: ¿cuál es el tratamiento que se da en la transición del exponente natural al exponente entero y racional, trabajados en algunos libros de texto de grado noveno? Donde se puede evidenciar la poca importancia dada a la jerarquía en las definiciones del concepto de potenciación y la escasa coherencia establecida en los textos escolares en el cumplimiento de las propiedades de los exponentes, lo cual marca una pauta importante en la identificación de las dificultades de nuestros estudiantes al abordar el exponente no natural.

\section{Fuentes}

Entre las principales fuentes se encuentra el análisis de textos de secundaria realizado por Martínez y Penalva (2006), donde se indaga por los procesos de simbolización del concepto de potencia; la caracterización de la convención matemática como un mecanismo de construcción de conocimiento, el caso de su funcionamiento en los exponentes, tesis doctoral de Martínez (2003) y los procesos de convención matemática como generadores de conocimiento. Entre las fuentes bibliográfi- cas se encuentran otros 28 títulos y 23 citas de pie de página que soportan los argumentos de esta tesis de maestría.

\section{Contenidos}

El documento de la tesis consta de seis capítulos. En el primero se encuentra el planteamiento del problema. En el segundo capítulo, Marco Teórico, se revisan las herramientas conceptuales utilizadas para alcanzar el objetivo de investigación. En el tercero, Metodología, se describe las estrategias que han llevado a cabo la realización de este trabajo. El cuarto capítulo, Análisis descriptivo del concepto de exponente, se presentan los hallazgos que se desprenden del análisis de los contenidos sobre el desarrollo del concepto de exponente no natural. El quinto, resume los principales resultados de esta investigación y en las recomendaciones y sugerencias para un trabajo futuro se presentan los elementos principales que podrían tener investigaciones basadas en ésta, así como algunas sugerencias a tener en cuenta para las editoriales colombianas de textos matemáticos con respecto al tratamiento que se dé al concepto de exponente. $Y$ las referencias. 


\section{Metodología}

El recorrido metodológico para la realización de este trabajo se puede acuñar al diseño que propone Alan Bishop en Villarreal (p.65), para la caracterización de una investigación en el campo de la Educación Matemática. Este recorrido está dado por las indagaciones preliminares, la revisión bibliográfica, la selección de textos del área de matemáticas de las editoriales educativas colombianas, la recolección de la información, el análisis de la información, la elaboración o planteamiento las de conclusiones y recomendaciones así como la elaboración del trabajo final.

\section{Conclusiones}

La realización de este trabajo de grado sugiere el planteamiento de cinco conclusiones sobre el análisis descriptivo del contenido estudiado, una sugerencia para futuros trabajos de grado y una recomendación a futura publicaciones sobre el tema de los exponentes en su representación natural, entera y racional.

Titulo. Elementos de análisis en la reprobación del aprendizaje del cálculo integral. Un estudio con estudiantes de mecánica automotriz.

\section{Autores. CARREÑO DÍAZ Andrés Julián y LEURO GIRALDO Alejandro David}

Palabras clave. Reprobación en Cálculo Integral, Clase de Matemáticas, Modelación, Tareas y Actividades Matemáticas, Normas Socio Matemáticas.

\section{Descripción}

Los altos índices de reprobación que se han presentado en la asignatura de Matemáticas II en el programa de Mecánica Automotriz de la Escuela Colombiana de Carreras Industriales (ECCI) donde se desarrolla el curso de Cálculo Integral, no son diferentes a los observados en otros estudios realizados mundialmente, los cuales se ubican en un intervalo de entre el 50\% y $80 \%$ (Mora, 2006, García 2006). El presente trabajo discute sobre el problema de reprobación en la asignatura de cálculo, y se centra en describir la clase de matemáticas II de la ECCI para analizar las relaciones entre algunos de sus componentes y establecer su relación con la reprobación.

\section{Contenido}

En el Capítulo 1 de la investigación se describe el planteamiento y antecedentes del problema desde una perspectiva social, con los objetivos propuestos; a continuación en el Capítulo 2, se abordan los elementos conceptuales y referenciales necesarios para el estudio, entre los que se encuentra 
la descripción de algunos elementos de la clase de matemáticas que permitieron realizar la interpretación y análisis de la información recolectada. En el Capitulo 3 se describe la metodología utilizada en la investigación, sus fases, técnicas para la recolección de información y las categorías que permiten caracterizar la reprobación en tres estudiantes de Mecánica Automotriz. Por último en los Capítulos 4 y 5 , se presentan los análisis e interpretación de la información recolectada.

\section{Metodología}

La investigación utilizó un enfoque metodológico cualitativo e interpretativo, y el método etnográfico centrado en un estudio de casos como herramienta para estudiar y comprender la clase de Matemáticas II a partir de las ideas, creencias, presupuestos, comportamientos y actitudes que tienen tanto estudiantes como profesor.

\section{Conclusiones}

Coincidimos con lo planteado por García (2006) y Alemán et. al, (2007) frente a la reprobación en los cursos del Cálculo Integral, la cual está determinada por multiplici- dad de factores que no se logran identificar de manera aislada, sólo el seguimiento sistemático y el conocimiento de cada población permitirá determinar las variables particulares asociadas a ésta.

Dentro de los factores relevantes presentes en la clase de Matemáticas II de la ECCl, pudimos establecer que las tareas matemáticas propuestas por el profesor a los estudiantes, aunque pueden promover la participación y comunicación en clase, no garantizan que los estudiantes acaten de manera eficiente las normas sociales, matemáticas y socio matemáticas establecidas en la clase, ya que el cumplimiento de éstas también dependen de la motivación y expectativas del estudiante, situación que influye con la reprobación de algunos estudiantes.

A partir de las encuestas realizadas a los estudiantes se logró evidenciar que era bastante motivante para ellos el aprendizaje del cálculo integral cuando tenían un empleo que se encontraba relacionado con la carrera técnica que estaban cursando, como en el caso del estudiante E3 que manifestaba la utilidad del cálculo en su desempeño profesional, a corto y mediano plazo. 
Título. Matemáticas y loncheras saludables: Un ambiente de aprendizaje de exploración e indagación relativo a situaciones multiplicativas con estudiantes de cuarto grado de primaria.

Autoras: PARRA DÍAZ, Diana Helena y ROJAS LEÓN, Jenny Carolina.

Palabras clave. Escenario de aprendizaje, ambientes de aprendizaje, escenarios de investigación, referencia, situaciones multiplicativas, loncheras saludables.

\section{Descripción}

Teniendo en cuenta que algunos estudios, reflejan las dificultades de los niños en la resolución de problemas con situaciones multiplicativas y al asociar los problemas de la clase de matemáticas y los de su cotidianidad; se presenta la creación de ambientes de aprendizaje teniendo en cuenta a Skovsmose (2000) con referencia en loncheras saludables de situaciones multiplicativas.

Se pretende que los estudiantes con el ambiente propuesto interpreten situaciones multiplicativas contextualizando su respuesta numérica de acuerdo con la situación, actúe en la situación social tomando decisiones, explore e indague en las actividades y el ambiente de clase sea una microsociedad democrática (Frankestein, 2009); características propias de la alfabetización matemática definida por Skovsmose (2000).

\section{Contenidos}

En el trabajo se encuentran cuatro capítulos; en el primero se hace una revisión de antecedentes (Estructura Multiplicativa y Escenarios de aprendizaje), se describe el problema, se plantean las preguntas de investigación y objetivos. En el segundo capítulo se presenta el marco de referencia teniendo en cuenta los aportes en la educación de la matemática crítica (Frankestein, 2009), ambientes de aprendizaje (Skovs- mose, 2000) y estructura multiplicativa (Vergnaud, 1999). En el tercero, se describe la metodología de investigación y los recursos utilizados para el diseño del ambiente de aprendizaje referenciado en loncheras saludables en situaciones multiplicativas y el sistema de categorías de análisis, teniendo en cuenta la alfabetización matemática (Skovsmose, 2000). Finalmente, el análisis de las evidencias, resaltando la labor del estudiante como sujeto activo de su aprendizaje dentro del ambiente.

\section{Metodología}

El enfoque utilizado fue el de Investigación acción. La muestra intencional del estudio se hizo con 12 estudiantes, entre los 8 y 9 años, de género masculino de cuarto grado de primaria. Los instrumentos que se utilizaron fueron: estudio piloto - gafas matemáticas, test escrito presentado en tira cómica, test escrito presentado en receta de cocina, cuento y elaboración de carta a modo de reflexión.

\section{Conclusiones}

Los resultados de la investigación muestran que bajo un ambiente de aprendizaje de exploración e indagación, los estudiantes relacionan su conocimiento matemático con problemas que deben resolver en su cotidianidad, recurriendo a procedimientos 
como la multiplicación y la suma repetitiva para tomar decisiones que conlleven a una vida más sana y justa, en su alimentación.

La aplicación de ambientes de aprendizaje en el aula con temas de interés para los estudiantes generan motivación hacia las actividades, además se encuentran actitudes de toma de decisiones cuando la situación lo requiere, teniendo un criterio personal utilizando las matemáticas y sus gustos en la comida. 